\author{
IN FUNCTIONAL EQUATIONS AND INEQUALITIES \\ BANACH CENTER PUBLICATIONS, VOLUME 99 \\ INSTITUTE OF MATHEMATICS \\ POLISH ACADEMY OF SCIENCES \\ WARSZAWA 2013
}

\title{
GENERALIZED MIDCONVEXITY
}

\author{
JACEK TABOR \\ Institute of Computer Science, Jagiellonian University \\ Łojasiewicza 6, 30-348 Kraków, Poland \\ E-mail: tabor@ii.uj.edu.pl \\ JÓZEF TABOR \\ Institute of Mathematics, University of Rzeszów \\ Rejtana 16A, 35-310 Rzeszów, Poland \\ E-mail:tabor@univ.rzeszow.pl \\ KRZYSZTOF MISZTAL \\ Institute of Computer Science, Jagiellonian University \\ Łojasiewicza 6, 30-348 Kraków, Poland \\ E-mail:krzysztof.misztal@ii.uj.edu.pl
}

\begin{abstract}
There are many types of midconvexities, for example Jensen convexity, $t$-convexity, $(s, t)$-convexity. We provide a uniform framework for all the above mentioned midconvexities by considering a generalized middle-point map on an abstract space $X$.

We show that we can define and study the basic convexity properties in this setting.
\end{abstract}

1. Introduction. Convexity has found applications in many parts of science. The simplest version of convexity is Jensen convexity (called also midconvexity). One can enumerate the following types of midconvexities:

(i) classical convexity in a linear space [9],

(ii) $t$-convexity 7 ,

(iii) $(s, t)$-midconvexities [7, 8],

(iv) $(M, N)$-convexity [6],

(v) metric convexity [1, 2],

(vi) convexity in Abelian groups (in particular in $\mathbb{Z}^{N}$ ) [3].

2010 Mathematics Subject Classification: Primary 26A51, 39B62; Secondary 26B25.

Key words and phrases: midconvex set, midconvex function, midconvex hull of a set. The paper is in final form and no version of it will be published elsewhere. 
We are going to unify and study the above mentioned midconvexities in one abstract theory. From now we assume that $X$ is a nonempty set with multivalued operation

$$
m_{X}: X \times X \multimap X,
$$

with possibly empty values, which satisfies

$$
x \in m_{X}(x, x) \text { for } x \in X .
$$

We interpret $m_{X}(x, y)$ as the set of middle-points between $x$ and $y$. If there is no doubt which middle-point function we have in mind we will write $m$ instead of $m_{X}$.

ExAmple 1.1. Commonly used middle-point functions:

(i) $X$ is a real vector space

- $m(x, y)=\{t x+(1-t) y\}$, where $t \in(0,1)$ is fixed;

- $m(x, y)=[x, y]=\{t x+(1-t) y: t \in[0,1]\}$;

- $m(x, y)=\left\{t^{s} x+(1-t)^{s} y\right\}$, where $t \in(0,1)$ and $s \in[1, \infty)$ are fixed;

- $m(x, y)=\left\{t^{s} x+(1-t)^{s} y: t \in[0,1]\right\}$, where $s \in[1, \infty)$ is fixed;

(ii) $(X, d)$ is a metric space

- $m(x, y)=\{z \in X: d(x, z)=t d(x, y) ; d(z, y)=(1-t) d(x, y)\}$, where $t \in[0,1]$ is fixed;

- $m(x, y)=\{z \in X: d(x, z)+d(z, y)=d(x, y)\}$

(iii) $(X,+)$ is an Abelian group

- $m(x, y)=\{z \in X: 2 z=x+y\}$;

(iv) $(X, \leq)$ is a linearly ordered set

- $m(x, y)=\{\min (x, y)\}$;

- $m(x, y)=\{\max (x, y)\}$

- $m(x, y)=\{z: \min (x, y) \leq z \leq \max (x, y)\}$

(v) $X=I$ where $I$ is a subinterval of $\mathbb{R}$ and $m(x, y)=\left\{M_{I}(x, y)\right\}$, where $M_{I}$ is a mean on $I$.

Using middle-point function we will define midconvex set and prove some its characteristic properties. Our aim is to show that one can build a natural convexity theory based on middle-point functions. In particular we will present the method of determining midconvex hull of a given set. In the last section we will define midconvex functions.

2. Midconvex sets. In this section we show how to define the natural convexity notions based on the middle-point functions. We begin with the definition of convex set.

Definition 2.1. We say that a set $W \subset X$ is $m_{X}$-convex if

$$
m_{X}(a, b) \subset W \text { for } a, b \in W .
$$


Condition (2) can be rewritten as follows

$$
m_{X}(W \times W) \subset W .
$$

Evidently the set $X$ is $m_{X}$-convex.

EXAMPLE 2.2.

(i) $X=\mathbb{R}, m(x, y)=\left\{\frac{x+y}{2}\right\}$. Then the set $\mathcal{D}$ of dyadic numbers is $m$-convex.

(ii) $X=(0, \infty), m(x, y)=\{\sqrt{x y}\}$. Then the set of positive algebraic numbers is $m$-convex.

Now we present some direct consequences of Definition 2.1.

PROPOSITION 2.3. Intersection of a nonempty family of $m$-convex sets is $m$-convex.

Proof. Let $\left\{W_{i}\right\}_{i \in I}$ be a nonempty family of $m$-convex sets. Consider arbitrary $a, b \in$ $\bigcap_{i \in I} W_{i}$. Then

$$
m(a, b) \subset W_{i} \text { for } i \in I,
$$

and hence

$$
m(a, b) \subset \bigcap_{i \in I} W_{i} .
$$

Proposition 2.4. Let $\left\{W_{i}\right\}_{i \in I}$ be a nonempty family of $m$-convex sets directed with respect to inclusion, i.e. such that for each $i_{1}, i_{2} \in I$ there exists $i_{3} \in I$ with the property $W_{i_{1}} \subset W_{i_{3}}, W_{i_{2}} \subset W_{i_{3}}$. Then

$$
W:=\bigcup_{i \in I} W_{i}
$$

is m-convex.

Proof. Consider arbitrary $a, b \in W$ and choose $i_{1}, i_{2} \in I$ such that $a \in W_{i_{1}}, b \in W_{i_{2}}$. Then we can find $i_{3} \in I$ such that

$$
a \in W_{i_{1}} \subset W_{i_{3}}, \quad b \in W_{i_{2}} \subset W_{i_{3}},
$$

whence we obtain

$$
m_{X}(a, b) \subset W_{i_{3}} \subset W .
$$

Suppose that we are given a set $Y \subset X$. In a natural way we define the restriction $m_{X \mid Y}: Y \times Y \multimap Y$ of $m_{X}$ to $Y$ by the formula

$$
m_{X \mid Y}\left(y_{1}, y_{2}\right):=m_{X}\left(y_{1}, y_{2}\right) \cap Y \text { for } y_{1}, y_{2} \in Y .
$$

One can easily observe that $m_{X \mid Y}$ is a middle-point map on $Y$.

Proposition 2.5. Let $W \subset Y \subset X$. If $Y$ is an $m_{X}$-convex subset of $X$ and $W$ is an $m_{X \mid Y}$-convex subset of $Y$ then $W$ is $m_{X}$-convex.

Proof. Consider arbitrary $w_{1}, w_{2} \in W$. Since $W$ is $m_{X \mid Y}$-convex

$$
m_{X \mid Y}\left(w_{1}, w_{2}\right)=m_{X}\left(w_{1}, w_{2}\right) \cap Y \subset W .
$$

However, by the $m_{X}$-convexity of $Y$ and the fact that $w_{1}, w_{2} \in W \subset Y$ we obtain that

$$
m_{X}\left(w_{1}, w_{2}\right) \subset Y .
$$

Consequently we obtain that $m_{X}\left(w_{1}, w_{2}\right)=m_{X}\left(w_{1}, w_{2}\right) \cap Y=m_{X \mid Y}\left(w_{1}, w_{2}\right) \subset W$. 
Assume that we are given nonempty sets $X, Y$ and $m_{X}$ and $m_{Y}$ satisfying (1). In the Cartesian product $X \times Y$ we define the middle-point operation $m_{X \times Y}$ by

$$
m_{X \times Y}\left(\left(x_{1}, y_{1}\right),\left(x_{2}, y_{2}\right)\right):=m_{X}\left(x_{1}, x_{2}\right) \times m_{Y}\left(y_{1}, y_{2}\right) \text { for } x_{1}, x_{2} \in X, y_{1}, y_{2} \in Y \text {. }
$$

Proposition 2.6. We assume that we are given nonempty sets $X, Y$ and operations $m_{X}, m_{Y}$ satisfying (1). Let $\emptyset \neq V \subset X$ and $\emptyset \neq W \subset Y$. Then $V \times W$ is $m_{X \times Y}$-convex if and only if $V$ is $m_{X}$-convex and $W$ is $m_{Y}$-convex.

Proof. Assume that $V$ is $m_{X}$-convex and $W$ is $m_{Y}$-convex. Consider arbitrary $\left(v_{1}, w_{1}\right)$, $\left(v_{2}, w_{2}\right) \in V \times W$. Then $m_{X}\left(v_{1}, v_{2}\right) \subset V$ and $m_{Y}\left(w_{1}, w_{2}\right) \subset W$, and consequently

$$
m_{X \times Y}\left(\left(v_{1}, w_{1}\right),\left(v_{2}, w_{2}\right)\right)=m_{X}\left(v_{1}, v_{2}\right) \times m_{Y}\left(w_{1}, w_{2}\right) \subset V \times W,
$$

which proves that $V \times W$ is $m_{X \times Y}$-convex.

Assume now that $V \times W$ is $m_{X \times Y}$-convex. Consider arbitrary $v_{1}, v_{2} \in V, w \in W$. Then

$$
m_{X \times Y}\left(\left(v_{1}, w\right),\left(v_{2}, w\right)\right)=m_{X}\left(v_{1}, v_{2}\right) \times m_{Y}(w, w) \subset V \times W,
$$

and hence $m_{X}\left(v_{1}, v_{2}\right) \subset V$, which proves that $V$ is $m_{X}$-convex. By the similar argumentation we obtain that $W$ is $m_{Y}$-convex.

Definition 2.7. Let $W \subset X$. Intersection of all $m_{X}$-convex sets containing $W$ is called $m_{X}$-convex hull of $W$ and denoted by $m_{X} \operatorname{conv}(W)$.

Since $X$ is $m$-convex it follows from Proposition 2.3 that the definition of $m_{X} \operatorname{conv}(W)$ is well-posed. It is the smallest $m_{X}$-convex set containing $W$.

To characterize $\operatorname{conv}(W)$ we define the sequence of sets. We put

$$
\begin{aligned}
m_{X} \operatorname{conv}_{0}(W) & =W, \\
m_{X} \operatorname{conv}_{1}(W) & =m_{X}(W \times W), \\
m_{X} \operatorname{conv}_{n+1}(W) & =m_{X} \operatorname{conv}_{1}\left(m_{X} \operatorname{conv}_{n}(W)\right) \text { for } n \in \mathbb{N}_{0}=\mathbb{N} \cup\{0\} .
\end{aligned}
$$

Theorem 2.8. Let $W \subset X$. Then

$$
m_{X} \operatorname{conv}(W)=\bigcup_{n \in \mathbb{N}_{0}} m_{X} \operatorname{conv}_{n}(W) .
$$

Proof. We first show that $\bigcup_{n \in \mathbb{N}_{0}} m_{X} \operatorname{conv}_{n}(W)$ is $m_{X}$-convex. Since it clearly contains $W$, this would prove by definition the inclusion $m_{X} \operatorname{conv}(W) \subset \bigcup_{n \in \mathbb{N}_{0}} m_{X} \operatorname{conv}_{n}(W)$.

Consider arbitrary $x, y \in \bigcup_{n \in \mathbb{N}_{0}} m_{X} \operatorname{conv}_{n}(W)$. Since the sequence $\left(m_{X} \operatorname{conv}_{n}(W)\right)_{n \in \mathbb{N}_{0}}$ is ascending, there exists $n \in \mathbb{N}_{0}$ such that $x, y \in m_{X} \operatorname{conv}_{n}(W)$, and consequently $m_{X}(x, y) \subset m_{X}\left(m_{X} \operatorname{conv}_{n}(W)\right)=m_{X} \operatorname{conv}_{n+1}(W)$.

Now we prove the converse inclusion. Since $m_{X} \operatorname{conv}(W)$ is $m$-convex and $W \subset$ $m_{X} \operatorname{conv}(W)$ we obtain that

$$
m_{X} \operatorname{conv}_{1}(W) \subset m_{X} \operatorname{conv}(W),
$$

and consequently that

$$
m_{X} \operatorname{conv}_{n}(W) \subset m_{X} \operatorname{conv}(W) \text { for } n \in \mathbb{N}_{0} .
$$

The last inclusion implies that $\bigcup_{n \in \mathbb{N}_{0}} \operatorname{conv}_{n}(W) \subset m_{X} \operatorname{conv}(W)$. 
As a direct corollary from Theorem 2.8 we obtain the following result.

Corollary 2.9. If $X$ is finite then there exists an $n_{0} \in \mathbb{N}_{0}$ such that

$$
m_{X} \operatorname{conv}_{n_{0}+1}(W)=m_{X} \operatorname{conv}_{n_{0}}(W) .
$$

Then

$$
m_{X} \operatorname{conv}(W)=m_{X} \operatorname{conv}_{n_{0}}(W) .
$$

We illustrate the above considerations.

EXAmPLE 2.10. Let $X=\mathbb{R}^{2}$ with the norm $\|(x, y)\|=\max (|x|,|y|)$, let $m$ be defined as follows

$$
\begin{aligned}
& m\left(\left(x_{1}, y_{1}\right),\left(x_{2}, y_{2}\right)\right)=\left\{(x, y) \in \mathbb{R}^{2}:\right. \\
& \left.\left\|(x, y)-\left(x_{1}, y_{1}\right)\right\|=\left\|(x, y)-\left(x_{2}, y_{2}\right)\right\|=\frac{1}{2}\left\|\left(x_{1}-x_{2}, y_{1}-y_{2}\right)\right\|\right\},
\end{aligned}
$$

and let $W=\{(0,0),(1,0)\}$. Then $\operatorname{conv}_{1}(W)$ and $\operatorname{conv}_{2}(W)$ are presented in Figure 1(a) and $1(\mathrm{~b})$.

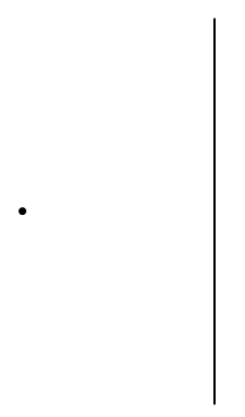

(a)

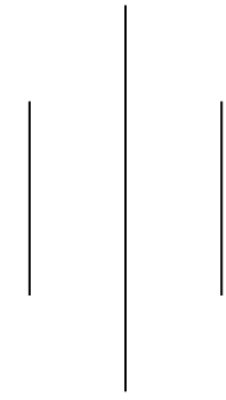

(b)

Fig. 1. $\operatorname{conv}_{1}(W)$ and $\operatorname{conv}_{2}(W)$

Moreover, we have

$$
\begin{aligned}
& m \operatorname{conv}(W)=\left\{(x, y) \in \mathbb{R}^{2}:\right. \\
& \left.\quad\left(x \in\left[0, \frac{1}{2}\right] \cap \mathcal{D}, y \in[-x, x]\right) \vee\left(x \in\left[\frac{1}{2}, 1\right] \cap \mathcal{D}, y \in[x-1,1-x]\right)\right\},
\end{aligned}
$$

where $\mathcal{D}$ denotes the set of dyadic numbers.

ExAmPLE 2.11. Let $X=\left(\mathbb{R}^{2}, \prec\right)$, where $\alpha$ is the lexicographic order, i.e.

$$
\left(x_{1}, y_{1}\right) \prec\left(x_{2}, y_{2}\right) \Longleftrightarrow x_{1}<x_{2} \vee\left(x_{1}=x_{2} \wedge y_{1} \leq y_{2}\right),
$$

let $m$ be defined as follows

$$
m\left(\left(x_{1}, y_{1}\right),\left(x_{2}, y_{2}\right)\right)=\left\{(x, y) \in \mathbb{R}^{2}:\left(x_{1}, y_{1}\right) \prec(x, y) \prec\left(x_{2}, y_{2}\right)\right\},
$$

and let $W=\{(0,0),(1,0)\}$. Then $\operatorname{conv}(W)=\operatorname{conv}_{1}(W)$ and it is presented at Figure2.

Now we define the notion of extreme point. 


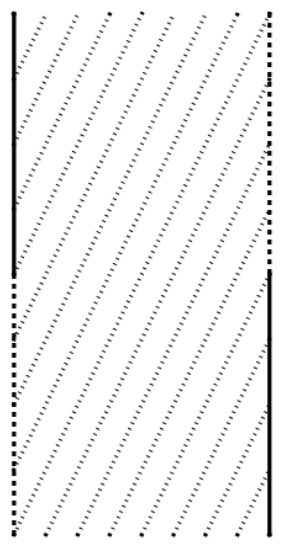

Fig. 2.

Definition 2.12. Let $W \subset X$ be an $m$-convex set. We say that $a \in W$ is an extreme point of $W$ if

$$
\forall x, y \in W \quad a \in m(x, y) \Rightarrow a=x \text { or } a=y .
$$

The set of extreme points of $m$-convex set $W$ will be denoted be ext $W$.

REMARK 2.13. Let $W \subset X$ be an $m$-convex set. One could consider another definition of extreme point by considering elements $a \in W$ for which

$$
\forall x, y \in W \quad a \in m(x, y) \Rightarrow a=x=y .
$$

However, then for the $m$ defined in Example 1.1 (first point) the extreme points would not coincide with the classical case.

More precisely, as was mentioned by the referee, for $X=\mathbb{R}, m(x, y)=[x, y]$ and $W=[0,1]$, we obtain that 0 is an extreme point of $[0,1]$, but the condition $(3)$ is not satisfied: for $x=0, y=1$ we have $0 \in m(0,1)$ but $0 \neq 1$.

It occurs that contrary to our intuition even finite sets may have no extreme points. EXAmple 2.14. Consider the set $\mathbb{Z}_{3}=\{0,1,2\}$ with addition modulo 3 and the middlepoint function defined by

$$
m(k, l)=\{j: 2 j=k+l\} .
$$

Then $0 \in m(1,2), 1 \in m(0,2), 2 \in m(0,1)$ and hence $\mathbb{Z}_{3}$ has no extreme points.

3. Midconvex functions. We are going to define the notion of midconvex function. For this purpose we need a specific class of means in $\mathbb{R}$. By $\mathcal{M}$ we denote the set of all functions $M: \mathbb{R} \times \mathbb{R} \rightarrow \mathbb{R}$ satisfying

$$
M(x, x)=x \text { for } x \in X
$$

and the condition

$$
\forall x_{1}, x_{2}, y_{1}, y_{2} \in \mathbb{R} \quad x_{1} \leq x_{2}, y_{1} \leq y_{2} \Rightarrow M\left(x_{1}, y_{1}\right) \leq M\left(x_{2}, y_{2}\right) .
$$


For a function $f: W \rightarrow \mathbb{R}$ by epi $f$ we denote its epigraph, i.e. we put

$$
\text { epi } f:=\{(x, y) \in W \times \mathbb{R}: y \geq f(x)\} .
$$

Definition 3.1. Let $W \subset X$, let $m_{X}$ be a middle-point function in $X$, and let $M \in \mathcal{M}$. We say that a function $f: W \rightarrow \mathbb{R}$ is $\left(m_{X}, M\right)$-convex if epi $f$ is $m_{X} \times\{M\}$-convex.

Proposition 3.2. Let $W \subset X$, let $m_{X}$ be a mean in $X$, and let $M \in \mathcal{M}$. A function $f: W \rightarrow \mathbb{R}$ is $\left(m_{X}, M\right)$-convex if and only if the following conditions hold:

(i) $W$ is $m_{X}$-convex;

(ii) $f(w) \leq M\left(f\left(x_{1}\right), f\left(x_{2}\right)\right)$ for $x_{1}, x_{2}, w \in W, w \in M\left(x_{1}, x_{2}\right)$.

Proof. Let $f: W \rightarrow \mathbb{R}$ be an arbitrary function. Assume that epi $f$ is $\left(m_{X}, M\right)$-convex. Consider arbitrary $x_{1}, x_{2} \in W$. Then $\left(x_{1}, f\left(x_{1}\right)\right),\left(x_{2}, f\left(x_{2}\right)\right) \in$ epi $f$ and consequently

$$
\left(m_{X}\left(x_{1}, x_{2}\right), M\left(f\left(x_{1}\right), f\left(x_{2}\right)\right)\right) \subset \text { epi } f .
$$

Inclusion (5) implies that $m_{X}\left(x_{1}, x_{2}\right) \subset W$. We have proved (i).

Furthermore it results from (5) that

$$
\left(w, M\left(f\left(x_{1}\right), f\left(x_{2}\right)\right)\right) \in \text { epi } f \text { for } w \in m_{X}\left(x_{1}, x_{2}\right) .
$$

But it means that

$$
M\left(f\left(x_{1}\right), f\left(x_{2}\right)\right) \geq f(w) \text { for } w \in m_{X}\left(x_{1}, x_{2}\right) .
$$

Thus we have proved that condition (ii) is valid.

Assume now that conditions (i) and (ii) are satisfied. Consider arbitrary $\left(x_{1}, y_{1}\right)$, $\left(x_{2}, y_{2}\right) \in$ epi $f$. Then $x_{1}, x_{2} \in W$ and hence by (i)

$$
m_{X}\left(x_{1}, x_{2}\right) \subset W .
$$

We have to prove that

$$
\left(w, M\left(y_{1}, y_{2}\right)\right) \in \text { epi } f \text { for } w \in m_{X}\left(x_{1}, x_{2}\right),
$$

i.e.

$$
M\left(y_{1}, y_{2}\right) \geq f(w) \text { for } w \in m_{X}\left(x_{1}, x_{2}\right) .
$$

Consider an arbitrary $w \in m_{X}\left(x_{1}, x_{2}\right)$. We have

$$
y_{1} \geq f\left(x_{1}\right), \quad y_{2} \geq f\left(x_{2}\right),
$$

and hence by 4

$$
M\left(y_{1}, y_{2}\right) \geq M\left(f\left(x_{1}\right), f\left(x_{2}\right)\right),
$$

whence by (ii) we obtain

$$
M\left(y_{1}, y_{2}\right) \geq f(w)
$$

which completes the proof.

ExAmple 3.3. Let $X$ be a real vector space and let $t \in(0,1)$ be arbitrarily fixed. Taking $m_{X}: X \times X \rightarrow X$ defined by

$$
m_{X}\left(x_{1}, x_{2}\right)=\left\{t x_{1}+(1-t) x_{2}\right\}
$$

and $M: \mathbb{R} \times \mathbb{R} \rightarrow \mathbb{R}$ defined by

$$
M\left(y_{1}, y_{2}\right)=t y_{1}+(1-t) y_{2},
$$


by Proposition 3.2, we obtain that $\left(m_{X}, M\right)$-convexity of a function $f: W \rightarrow \mathbb{R}, W \subset X$ is equivalent to its $t$-convexity (in the classical sense). In particular for $t=\frac{1}{2}$ we obtain definition of the Jensen convex function.

If we take $m_{X}$ defined by

$$
m_{X}\left(x_{1}, x_{2}\right)=\left[x_{1}, x_{2}\right]=\left\{s x_{1}+(1-s) x_{2}: s \in[0,1]\right\},
$$

and $M$ defined by

$$
M\left(y_{1}, y_{2}\right)=\max \left(y_{1}, y_{2}\right)
$$

we obtain that $\left(m_{X}, M\right)$-convexity of a function is equivalent to its quasiconvexity (in the classical sense).

Convex function (in the classical sense) can be defined as $\left(m_{t}, m_{t}\right)$-convex for every $t \in[0,1]$, where

$$
m_{t}\left(x_{1}, x_{2}\right)=\left\{t x_{1}+(1-t) x_{2}\right\} .
$$

The next result shows that $\left(m_{X}, M\right)$-convex functions have similar properties as convex (midconvex) ones.

Proposition 3.4. Let $W \subset X$ be an $m_{X}$-convex set and let $M: \mathbb{R} \times \mathbb{R} \rightarrow \mathbb{R}$ be of the form

$$
M\left(x_{1}, x_{2}\right)=t x_{1}+(1-t) x_{2}
$$

for some $t \in[0,1]$. Then the family of all $\left(m_{X}, M\right)$-convex functions $f: W \rightarrow \mathbb{R}$ is closed under addition, multiplication by positive number and operation of supremum.

Proof. The proofs rely on direct applications of condition (ii) from Proposition 3.2. We present the proof for supremum.

Let $f_{i}: w \rightarrow \mathbb{R}, i \in I$, be any family of $\left(m_{X}, M\right)$-convex functions. By Proposition 3.2 we have for $i \in I, x_{1}, x_{2} \in W, w \in m_{X}\left(x_{1}, x_{2}\right)$

$$
f_{i}(w) \leq t f_{i}\left(x_{1}\right)+(1-t) f_{i}\left(x_{2}\right) .
$$

Hence we obtain for $x_{1}, x_{2} \in W, w \in m_{X}\left(x_{1}, x_{2}\right)$

$$
\begin{aligned}
\sup _{i \in I} f_{i}(w) & \leq \sup _{i \in I}\left(t f_{i}\left(x_{1}\right)+(1-t) f_{i}\left(x_{2}\right)\right) \\
& \leq t \sup _{i \in I} f_{i}\left(x_{1}\right)+(1-t) \sup _{i \in I} f_{i}\left(x_{2}\right) .
\end{aligned}
$$

It means that the function $\sup _{i \in I} f_{i}$ is $\left(m_{X}, M\right)$-convex.

\section{References}

[1] H. Ben-El-Mechaiekh, S. Chebbi, M. Florenzano, J. V. Llinares, Abstract convexity and fixed points, J. Math. Anal. Appl. 222 (1998), 138-150.

[2] K. Goebel, W. A. Kirk, Topics in Metric Fixed Point Theory, Cambridge Stud. Adv. Math. 28, Cambridge Univ. Press, Cambridge, 1990.

[3] W. Jarczyk, M. Laczkovich, Convexity on abelian groups, J. Convex Anal. 16 (2009), 33-48. 
[4] M. Kuczma, An Introduction to the Theory of Functional Equations and Inequalities. Cauchy's Equation and Jensen's Inequality, Prace Naukowe Uniwersytetu Śląskiego w Katowicach 489, Uniwersytet Śląski, Katowice; Państwowe Wydawnictwo Naukowe, Warszawa, 1985.

[5] K. Murota, Discrete Convex Analysis, SIAM Monogr. Discrete Math. Appl., Society for Industrial and Applied Mathematics (SIAM), Philadelphia, PA, 2003.

[6] C. Niculescu, L. Persson, Convex functions and their applications. A contemporary approach, CMS Books Math./Ouvrages de Math. SMC 23, Springer, New York, 2006.

[7] K. Nikodem, Z. Páles, On t-convex functions, Real Anal. Exchange 29 (2003/04), 219-228.

[8] W. Orlicz, A note on modular spaces. I, Bull. Acad. Polon. Sci. Sér. Sci. Math. Astronom. Phys. 9 (1961), 157-162.

[9] R. T. Rockafellar, Convex Analysis, Princeton Math. Ser. 28, Princeton Univ. Press, Princeton, N.J., 1970. 
